\title{
Electronic Structure of Iron-Based Superconductor Studied by EELS and CBED
}

\author{
C. Ma***, L. Wu*, H.X. Yang**, H.L. Shi**, Z.W. Wang**, and J.Q. Li**, and Y. Zhu* \\ * Department of Condensed Matter Physics, Brookhaven National Laboratory, Upton, New York, \\ 11973 \\ ** Beijing National Laboratory for Condensed Matter Physics, Institute of Physics, Chinese \\ Academy of Sciences, Beijing 100190, China
}

The discovery of the superconductivity in Fe-pnictide system has led to a resurgence of interest in superconductivity research because their transition temperatures up to $56 \mathrm{~K}$ are the highest except for cuprate superconductors [1,2]. Both experimental measurements and theoretical calculations indicate that several features of these materials are in sharp contrast to the cuprates. The quasi-twodimensional electronic properties in the cuprates led to the speculation that the reduced dimensionality is a prerequisite for superconductivity at temperature above $40 \mathrm{~K}$. However, it is not the case in iron pnictids, since the recent experimental measurements and theoretical calculations demonstrated strong $\mathrm{k}_{\mathrm{z}}$ dispersion and three-dimensional Fermi-surface topology [3]. Therefore, the interlayer hybridization between the insulating and conducting (FeAs) layers would play an important role in the dimensionality of the Fermi surface and orbital occupancy. Another issue which is still controversial in iron pnictides is the doping effect on the electronic structure [4]. The superconductivity can be induced by electron and hole doping in the insulating layers and transition metal doping on the Fe sites, which offers the experimental access to a wide set of materials with various electronic states.

We use a combination of electron energy loss spectroscopy (EELS), quantitative convergent beam electron diffraction (CBED) and density functional theory (DFT) calculations to characterize the electronic structure of the iron-based compounds, particularly focusing on the interlayer hybridization and doping effect. The EELS measurements and calculations (Fig. 1 and 2) indicate that the iron ions are in intermediate spin state and that the interlayer hybridization plays an important role in the bandwidth, orbital occupancy and magnetic moment. These results make iron pnictides quite unlike cuprate superconductors, and indicate that reduced dimensionality is not a necessary condition for searching high-Tc superconductors. In order to study the doping effect on the charge distribution and orbital-dependent modification of the electronic structure in Co-doped $\mathrm{Ba}(\mathrm{Fe}, \mathrm{Co})_{2} \mathrm{As}_{2}$, the quantitative CBED method was employed. With the low-index structure factors accurately measured by CBED (Fig. 3) and the median- and high-index structure factors calculated by DFT, the charge density and orbital occupancy can be retrieved through the mutipole refinement. The substitution of Co for Fe atoms doesn't introduce extra electrons into other Fe sites, that is, the valence state of Fe ions keep constant. However, the obtained orbital occupancy suggests electron redistribution among the Fe 3d orbitals, which is consistent with the EELS measurements [5].

\section{References}

[1] Y. Kamihara et al., J. Am. Chem. Soc. 130, 3296 (2008).

[2] Z.A. Ren et al., Europhys. Lett. 83, 17002 (2008).

[3] P. Vilmercati et al., Phys. Rev. B 79, 220503(R) (2009).

[4] H. Wadati et al., Phys. Rev. Lett. 105, 157004 (2010).

[5] Work supported by the U.S. DOE under contract DE-AC02-98CH10886. 


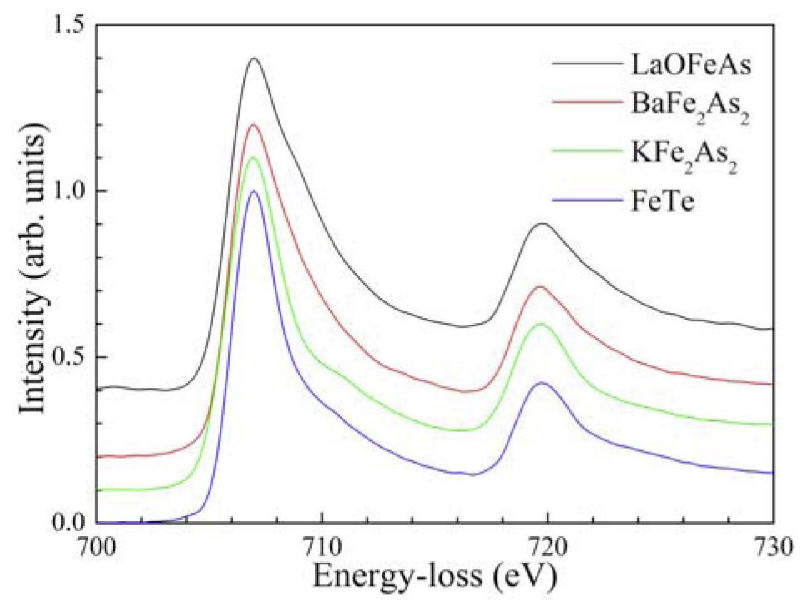

Fig. 1 Experimental $\mathrm{Fe} \mathrm{L}_{2,3}$ edges for $\mathrm{LaFeAsO}$, $\mathrm{BaFe}_{2} \mathrm{As}_{2}, \mathrm{KFe}_{2} \mathrm{As}_{2}$, and $\mathrm{FeTe}$ acquired under the same experimental conditions. These spectra show very similar features, but the branching ratios indicate the intermediate spin state of $\mathrm{Fe}$ ions and different magnetic moment for different layered structures. For the sake of clarity, the spectra were shifted in such a way that their maxima are aligned.

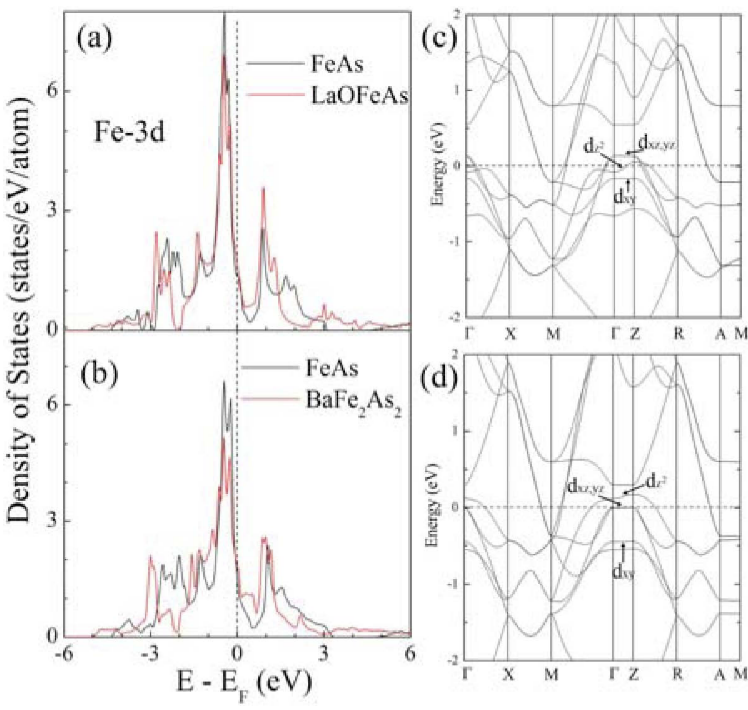

Fig. 2 Comparison of density of states of periodic FeAs layers with $\mathrm{LaFeAsO}$ (a) and $\mathrm{BaFe}_{2} \mathrm{As}_{2}$ (b). Electronic bandstructure in the vicinity of the Fermi level for $\mathrm{LaFeAsO}$ (c) and for corresponding periodic FeAs layers (d). The Fermi energy is set to zero. The difference between the solid $\mathrm{BaFe}_{2} \mathrm{As}_{2}$ and corresponding periodic FeAs layers suggests the interlayer hybridization leads to an interorbital charge transfer.
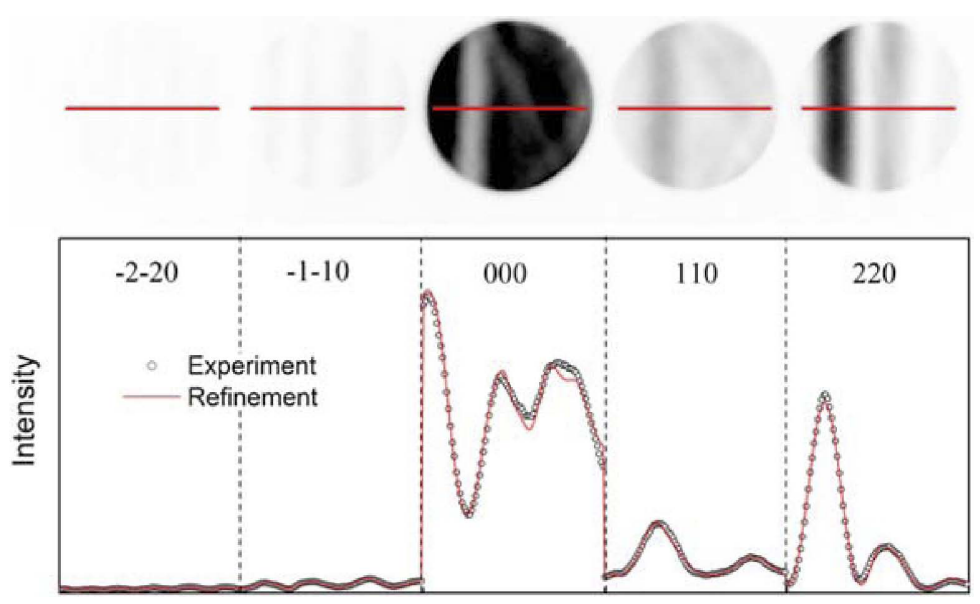

Fig. 3 Experimental energy-filtered CBED pattern of $\mathrm{BaFe}_{2} \mathrm{As}_{2}$ at 110 systematical row and line scan of the intensity profile from the experimental pattern. Open circles are experimental data and the red curve are calculated one after refinement of the structure factors. 